\title{
Damage Detection and Localisation in Buried Pipelines using Entropy in Information Theory ${ }^{\dagger}$
}

\author{
Rosario Ceravolo ${ }^{1}$, Marco Civera ${ }^{1}{ }^{*}$, Erica Lenticchia ${ }^{1}$, Gaetano Miraglia $^{1}$, and Cecilia Surace ${ }^{1}$ \\ 1 Politecnico di Torino, C. Duca Degli Abruzzi, 24, 10129 Turin, Italy; rosario.ceravolo@polito.it (R.C.); \\ erica.lenticchia@polito.it (E.L.), gaetano.miraglia@polito.it (G.M.), cecilia.surace@polito.it (C.S.) \\ * Correspondence: marco.civera@polito.it \\ † Presented at 1st International Electronic Conference on Applied Sciences, 10-30 November 2020; Available \\ online: https://asec2020.sciforum.net/.
}

Published: 10 November 2020

\begin{abstract}
In recent years, entropy measures, and more specifically, spectral entropy have emerged as an efficient method for the damage assessment of both mechanical systems and civil structures. In the present work, entropy measures are applied as a damage-sensitive feature for the real-time structural health monitoring of buried pipelines. The management of these underground Fluids Distribution Systems (FDSs) is critical for supplying clean water, oil, gas, and other goods. However, the health state of these systems tends to deteriorate over time so that they become more vulnerable to leaks or catastrophic failure events. Maintenance surveys and visual inspections are expensive and labour-intensive, due to the difficulties in accessing buried pipelines. Thus, Vibration-Based Inspection (VBI) techniques and continuous monitoring would be perfectly suited for the task. The approach is validated numerically on the soil-structure models of a typical pipeline structure (i.e. Steel Pipes - SPs).
\end{abstract}

Keywords: Steel Pipes; Pipeline Inspection Management; Oil \& Gas Engineering; Wiener Entropy; Structural Health Monitoring; Real-Time Monitoring; Damage Localisation; Information Theory

\section{Introduction}

Pipelines represent one of the most efficient ways to transport fluid products (liquids or gases); they are generally considered as safer, more sustainable, and more economically convenient than other alternatives such as road or rail transportation [1]. The main uses include the off- and on-shore movement of oil and gas commodities, the transportation of raw and refined goods in chemical plants or other industrial processes, and the urban pipeline networks. These latter include the ones employed for sewerage, the potable Water Distribution Systems (WDSs), and the gas distribution network.

Due to their need to transport fluids over long distances and/or in densely inhabited environments, most of the pipelines are buried underground, making it difficult to reach them for visual inspection. Even when they are relatively easy to be accessed, e.g. thanks to the presence of maintenance holes, visual and human-made surveys are not cost-efficient due to the length and ramifications of the network. Other common survey strategies, such as the use of Pipeline Inspection Gauge (PIG) [2], have their limitations. For instance, an instrumented PIG may remain stuck into the pipe and cause further disruptions. For several other reasons (e.g. a very small diameter of the pipeline or due to the transportation of explosive fluids), some pipelines may be unpiggable [3]. Therefore, automatic and continuous Structural Health Monitoring (SHM) approaches, especially vibration-based ones [4], would be preferable. Indeed, Pipeline Integrity Management (PIM) [5] is essential for all the applications enlisted above. Mechanical fatigue, corrosion, rapid or slow ground 
movements (such as landslides or long-term subsidence), and other natural or human-made phenomena can produce pointwise cracks and leakages. Manufacturing defects can also result in internal cavities which represent a weak point of the structure. The presence of these leaking cracks always results in an economic loss and potentially in dangerous scenarios. For a WDS, this can lead to indoor floods or water contamination. Oil and wastewater spills may cause environmental pollution. Finally, oil and gas leakage can result in explosions, which are particularly dangerous in urban areas with high population density. These problems are even worse in the case of pressurised pipelines, where the internal pressure can rapidly turn a small crack into more extensive damage [6].

Several techniques have been proposed in recent years by the authors to extract useful information from the dynamics of an investigated structure; the Teager-Kaiser Energy Cepstral Coefficients [7,8], the bispectrum [9], and the Wavelet Levels [10] are a few examples of DamageSensitive Features (DSFs). The Vibration-Based Investigation (VBI) of pipeline systems is not as common as for other applications (such as bridge monitoring), but there are some applications reported in the scientific literature. To cite an example, Bao et al. [11] studied numerically and experimentally the vibrational response of an offshore pipeline system model, applying an Autoregressive Moving Average (ARMA) model. Strain measurements performed via distributed fibre optic sensors were used in [12]. Some comprehensive reviews of vibrational and nonvibrational techniques can be found in [13]. In this context, a Wiener Entropy (WE)-based methodology, recently introduced by the authors in [14], is here applied for damage detection and localisation (corresponding to the level 1 and 2 of the Rytter's Hierarchy [15] of SHM techniques, respectively). Promising results were achieved on some numerical case studies. The rest of the paper is organised as follow. Section 2 briefly defines the context of Pipeline Integrity Management and the main typologies of pipelines. Section 3 describes the proposed methodology for damage detection and localisation. Section 4 defines the numerical case study. Section 5 presents the results, and conclusions follow.

\section{Buried Pipelines}

Three factors mainly define a pipeline: the diameter, external environment, and the construction material. The present work focuses on buried (i.e. underground) and relatively large diameters (more than $60 \mathrm{~cm}$ ), very common in WDSs. In these cases, two typologies of pipelines are mostly encountered: Steel Pipes (SP) and Pre-stressed Concrete Cylinder Pipes (PCCP). As their names imply, the difference lies principally in the construction material used. This design option affects the pipeline behaviour (e.g. its sensitivity to corrosion) noticeably. PCCPs are also known as "rigid" pipes; the loads are mostly carried by the structure of the pipeline itself, with minimal contribution from the surrounding soil. SPs are, on the other hand, referred to as "flexible" pipes, as the soil-pipe integrated system supports the mechanical loads. A more systematic discussion can be found in the book of Young \& Trott [16]. In many applications, SPs are generally preferred as they can deflect without breaking, are more lightweight, and more adaptable and/or modifiable thanks to welding; yet they have higher costs and need special protection from corrosion [17]. The typical failure mechanisms include pitting corrosion, blowout hole, thinning of the pipeline after corrosion and graphitization, and pipe burst due to transient pressure or the "water hammer" phenomenon. The most typical damages encountered in buried pipes can be classified according to their geometry as circumferential, longitudinal, bell splitting, corrosion pitting, spiral, and bell shearing cracks, plus blowout holes [18]. In the present paper, the case of damage due to persistent corrosion and environmental actions on a buried SP will be numerically analysed in detail.

\section{The Damage Detection Method}

According to the Information Theory, the entropy is a measure of the ignorance of the investigated system [19]. Claude E. Shannon introduced entropy as a measure of uncertainty in 1948 [20]. Since this first definition, many variations have been proposed, with slightly different mathematical and statistical properties. For the sake of this research, The Wiener Entropy (WE) has been utilised and applied on real-time to data from a simulated continuous monitoring system. The 
measure of WE (also known as spectral flatness) $H_{w}$, can be defined as the ratio of the geometric mean of the power spectrum over the arithmetic mean of the same, i.e.

$$
H_{w}=Q \frac{\sqrt[Q]{\prod_{q} S(q)}}{\sum_{q} S(q)}
$$

where $Q$ represents the total number of frequency bins, and $S(q)$ is the discrete power spectrum of recorded vibrational time history. The rationale for its use in SHM is relatively straightforward and derives from the last proposed Axiom of Structural Health Monitoring, added in [21] to the well-known initial group of seven [22]. The work of Farrar et al. [21] highlighted the potential for Information Theory-based approaches in SHM, by stating that "damage increases the complexity of a system" and it is thus detectable as an instantaneous, localised variation in the signal entropy. However, in [14], the authors show as spectral entropy measures can behave differently with respect to damage based on the chosen reference signal (e.g. displacement, strain or acceleration). For example, the spectral entropy evaluated starting from displacement measures would decrease in the presence of damage. It was also shown how the Wiener Entropy (WE) if compared to the more common Shannon Spectral Entropy (SSE), is affected by the alterations to the system state in a more significant way; making it more damage-sensitive and suitable for detecting smaller cracks at the beginning of their development. On the other hand, WE is also more sensitive to measurement noise and damage-unrelated effects, which hampers its use for applications such as masonry structures, where temperature and humidity effects are non-negligible. However, these same effects are less pronounced in more homogeneous materials such as steel. For these reasons, it is convenient to trade off some stability of the results for more sensitivity to damage and opt for WE over SSE.

\section{Numerical Case Study}

A 9.5 m-long section of a pipeline was modelled in Ansys Mechanical APDL; a 4-node (3 Degrees of Freedom - DoF per node) SOLID186 element (brick element) has been used to model the soil, while the SP has been discretised with a 4-node (6 DoFs per node) SHELL181 element. The FE model is portrayed in Figure 1.a. The size and geometry of the pipe were set accordingly to the requirements specified in BS 5911-1 [23]. Considering a thickness of $0.04 \mathrm{~m}$, it resulted in a diameter of $1.48 \mathrm{~m}$ for the innermost surface and $1.52 \mathrm{~m}$ for the outer side. The centroid of the pipe cross-section is $2.25 \mathrm{~m}$ below ground level. A sensitivity analysis was carried to size the volume of the surrounding soil to be modelled (defined by the radius $R_{e}$ ). $R_{e}=9.5 \mathrm{~m}$ was found as the minimal value that eliminates all possible calculation errors related to the presence of the boundary in the model. The sensitivity of the model to the boundary was evaluated by checking the axial strain values in the pipeline, triggered by a change of $R_{e}$. For values of $R_{e}$ greater than $9.5 \mathrm{~m}$ the axial strain due to self-weight remained unchanged. A set of 4 optic fibres (surrounding the pipeline in position $\theta=0^{\circ}, 90^{\circ}, 180^{\circ}, 270^{\circ}$ for a total monitored length of $9.5 \mathrm{~m}$; see Figure 1.b) was simulated for the output channels. The acquisition of axial strains (along the $\mathrm{Z}$ direction in the model) was discretised at steps of $0.50 \mathrm{~m}$, resulting in 44 measurement points.

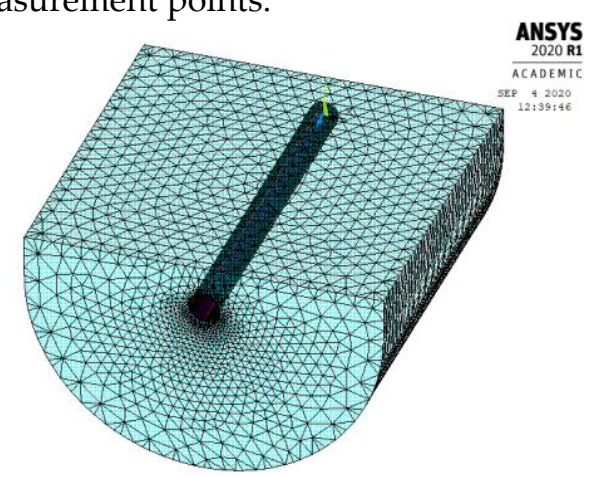

(a)

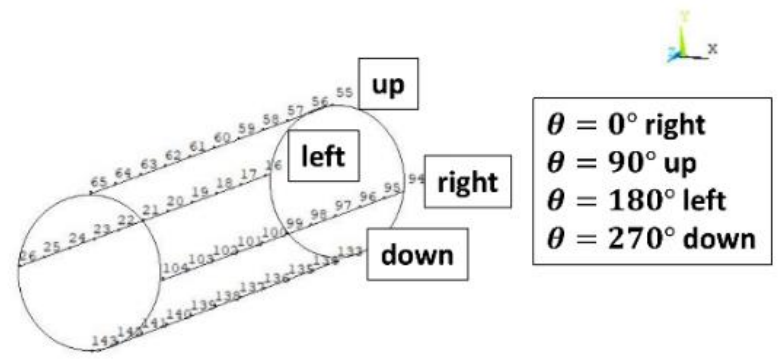

(b)

Figure 1. (a) Soil-Pipeline FE Model in Ansys Mechanical APDL; (b) assumed disposition of the optic fibres. 
Soil and steel properties are enlisted in Table 1 here below. The behaviour of steel was modelled as elastic, with standard properties values retrieved from [24]. The same consideration was applied for the soil since the analyses are supposed in operational conditions, with typical values again accordingly to [24]. The damage was modelled as a 50\% reduction of the pipe cross-section between $x=2.5$ and $3.0 \mathrm{~m}$, and $\theta$ between $0^{\circ}$ and $90^{\circ}$, by switching off the corresponding elements (thus, with a localised reduction of both mass and stiffness). The assumed damage configuration may be associated with sudden damage caused by persistent corrosion (e.g. flaking). A transient dynamic analysis was carried on both the undamaged and damaged model. The total duration of the simulations was set to $120 \mathrm{~s}$ for both models, with a sampling frequency of $1000 \mathrm{~Hz}$. In particular, two analyses have been performed to simulate the health and the damaged condition. Then, the predicted output (in terms of axial strain) have been concatenated in time to simulate a continuous monitoring over a time window of $120 \mathrm{~s}$ ( $60 \mathrm{~s}$ in health conditions and $60 \mathrm{~s}$ in damaged conditions). Thus, the damage is supposed to occur at $t_{0}=60 \mathrm{~s}$. A Gaussian noise with standard deviation equal to the $5 \%$ of the standard deviation of the signals output was applied to simulate noise in the sensors. A White Gaussian Noise (WGN) was instead applied to simulate input ambient vibrations.

Table 1. Mechanical properties of the FE models.

\begin{tabular}{ccc|ccc}
\hline \multicolumn{2}{c|}{ Soil } & & \multicolumn{3}{c}{ Steel } \\
\hline Density & 1850 & $\mathrm{~kg} / \mathrm{m}^{3}$ & Density & 7850 & $\mathrm{~kg} / \mathrm{m}^{3}$ \\
Young's Modulus & 24 & $\mathrm{MPa}$ & Young's Modulus & $210.7 \cdot 10^{3}$ & $\mathrm{MPa}$ \\
Poisson's Ratio & 0.20 & - & Poisson's Ratio & 0.30 & - \\
\hline
\end{tabular}

\section{Results}

Figure 2 and Figure 3 show the effects of the occurrence of damage in the pipeline and a comparison of this with the scenario without damage (for the same input), respectively. In the top left corner of each sub-figure, the location-time analysis of the recorded signal is reported in 2 dimensions (2D). The same information can be visualised in a 3D view in the bottom right quadrant. In the top right corner, the time projection shows the instant variation of WE induced by the damage occurrence. Finally, in the bottom left, the space projection localises the crack along the main pipeline axis (Z-coordinate). It can be noticed from Figure 2.a how the undamaged FE model suffered no localised and/or instantaneous variations of its WE distribution, which keeps on following a smooth curve along the whole inspected length and remains unchanged along time. On the other hand, the insertion of the damage induces a localised and instantaneous drop of the Wiener Entropy, as expected for a system of increasing structural non-uniformity. From Figure 3, the location and instant of occurrence of damage are clear for the two optic fibres deployed at $\theta=0^{\circ}$ and $90^{\circ}$, which correspond to the actual damage position.

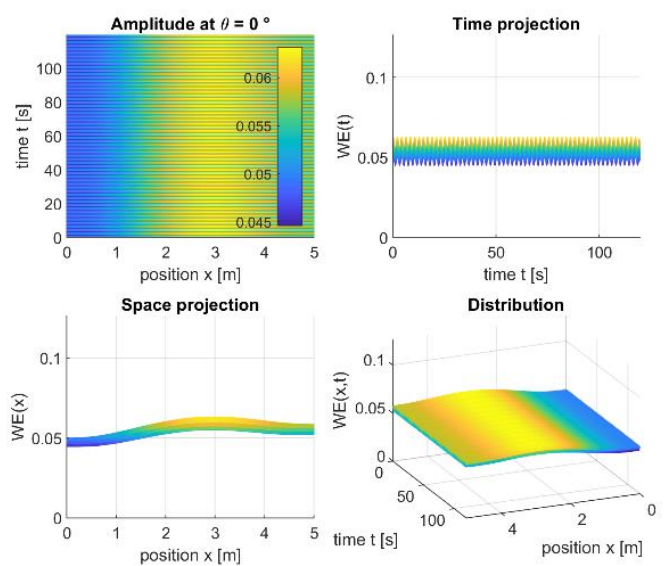

(a)

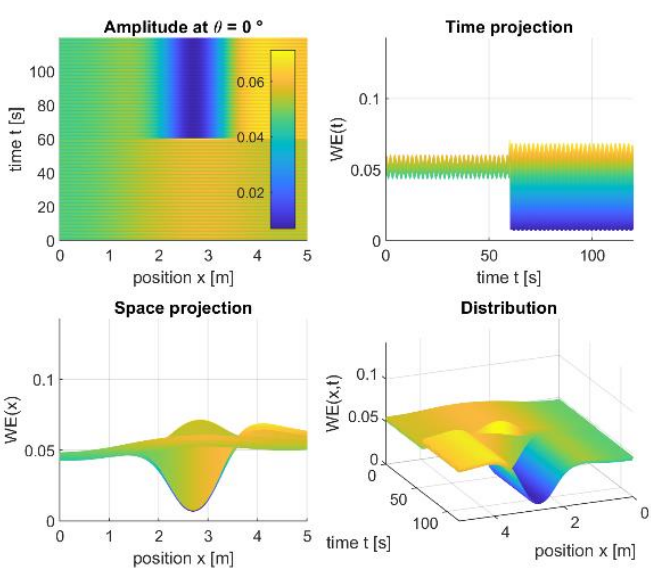

(b)

Figure 2. Comparison of the undamaged and damaged cases at $\theta=0^{\circ}$ (absence of noise). (a) undamaged pipeline; (b) damaged pipeline. 


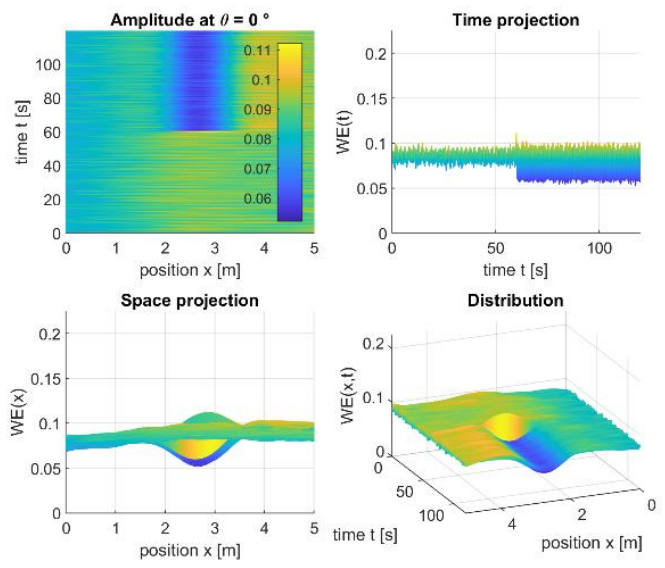

(a)
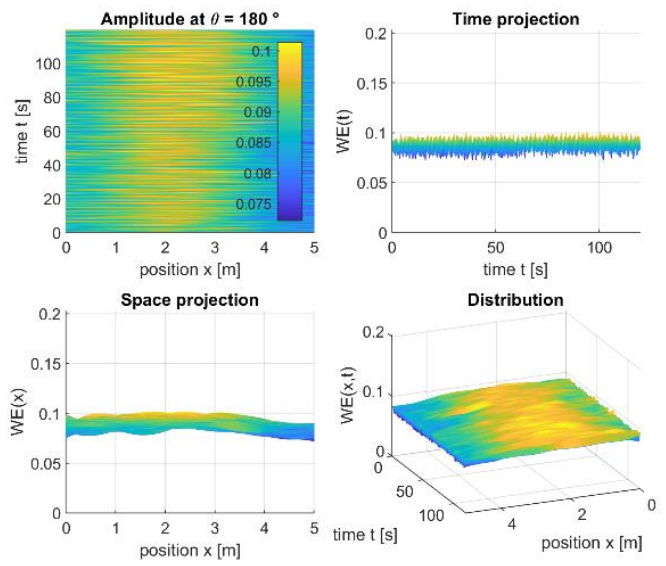

(c)
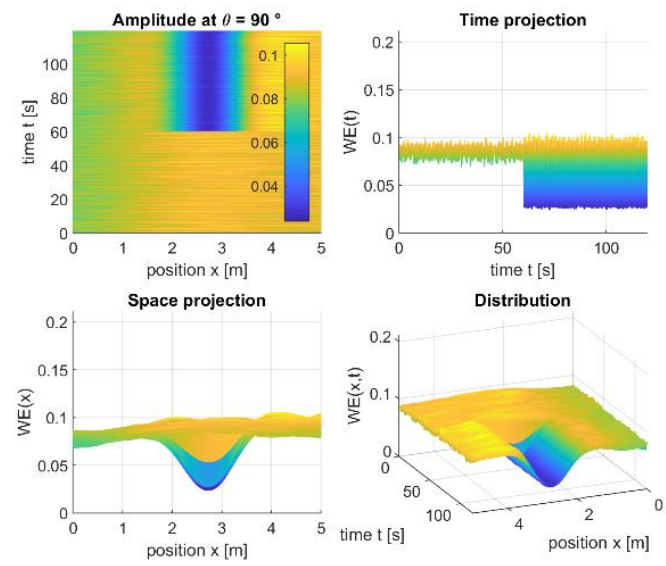

(b)
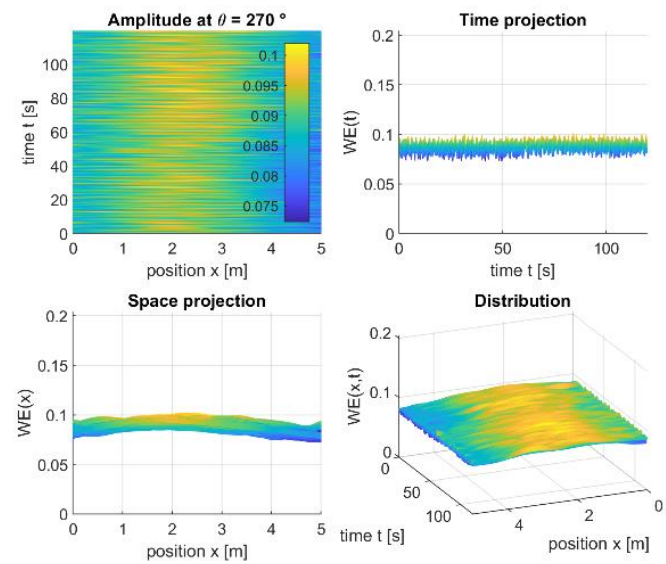

(d)

Figure 3. Damage analysis ( $5 \%$ of noise). (a) $\theta=0^{\circ}$; (b) $\theta=90^{\circ}$; (c) $\theta=180^{\circ}$; (d) $\theta=270^{\circ}$.

\section{Conclusions}

The damage detection method based on the WE variation proved to be a valid and promising approach for real-time monitoring of steel pipelines. It has been shown as WE is capable of providing information regarding the presence and the location of the damage. This method is in his first stage application and could be implemented in future research taking in consideration the variation of entropy associated to the severity of the damage (level 3 according to the Rytter's Hierarchy).

Author Contributions: conceptualization, G.M., R.C. and E.L.; methodology, G.M., R.C. and E.L.; software, G.M.; validation, G.M. and M.C.; resources, R.C.; data curation, G.M. and M.C.; writing-original draft preparation, M.C.; writing-review and editing, M.C. and G.M.; visualization, G.M. and M.C.; supervision, R.C. and C.S.; project administration, R.C. and C.S.

Funding: This research received no external funding.

Acknowledgements: The Authors would like to thank Mr Antonio Benedetto and prof Maria Pina Limongelli of Politecnico di Milano for their precious advice and collaboration.

Conflicts of Interest: The authors declare no conflict of interest.

\section{References}

1. Papadakis, G.A. Assessment of requirements on safety management systems in EU regulations for the control of major hazard pipelines. J. Hazard. Mater. 2000, 78, 63-89.

2. Quarini, J.; Shire, S. A Review of Fluid-Driven Pipeline Pigs and their Applications. Proc. Inst. Mech. Eng. Part E J. Process Mech. Eng. 2007, 221, 1-10.

3. Teitsma, A.; Takach, S.; Maupin, J.; Fox, J.; Shuttleworth, P.; Seger, P. Small diameter remote field eddy 
current inspection for unpiggable pipelines. In Proceedings of the Journal of Pressure Vessel Technology, Transactions of the ASME; American Society of Mechanical Engineers Digital Collection, 2005; Vol. 127, pp. 269-273.

4. Farrar, C.R.; Worden, K. Structural Health Monitoring: A Machine Learning Perspective; John Wiley \& Sons, L., Ed.; 2013;

5. Kishawy, H.A.; Gabbar, H.A. Review of pipeline integrity management practices. Int. J. Press. Vessel. Pip. 2010, 87, 373-380.

6. Chmelko, V.; Garan, M.; Šulko, M.; Gašparík, M. Health and Structural Integrity of Monitoring Systems: The Case Study of Pressurized Pipelines. Appl. Sci. 2020, 10, 6023.

7. Ferraris, M.; Civera, M.; Ceravolo, R.; Surace, C.; Betti, R. Using Enhanced Cepstral Analysis for Structural Health Monitoring. In; Springer, Singapore, 2020; pp. 150-165.

8. Civera, M.; Ferraris, M.; Ceravolo, R.; Surace, C.; Betti, R. The Teager-Kaiser Energy Cepstral Coefficients as an Effective Structural Health Monitoring Tool. Appl. Sci. 2019, 9, 5064.

9. Civera, M.; Zanotti Fragonara, L.; Surace, C. A novel approach to damage localisation based on bispectral analysis and neural network. Smart Struct. Syst. 2017, 20, 669-682.

10. Civera, M.; Zanotti Fragonara, L.; Surace, C. Nonlinear Dynamics of Cracked, Cantilevered Beam-like Structures Undergoing Large Deflections. In Proceedings of the metrology for aerospace; 2019.

11. Bao, C.; Hao, H.; Li, Z. Vibration-based structural health monitoring of offshore pipelines: numerical and experimental study. Struct. Control Heal. Monit. 2013, 20, 769-788.

12. Glisic, B.; Yao, Y. Fiber optic method for health assessment of pipelines subjected to earthquake-induced ground movement. Struct. Heal. Monit. An Int. J. 2012, 11, 696-711.

13. Liu, Z.; Kleiner, Y. State-of-the-art review of technologies for pipe structural health monitoring. IEEE Sens. J. 2012, 12, 1987-1992.

14. Ceravolo, R.; Lenticchia, E.; Miraglia, G. Spectral entropy of acceleration data for damage detection in masonry buildings affected by seismic sequences. Constr. Build. Mater. 2019, 210, 525-539.

15. Rytter, A. Vibrational Based Inspection of Civil Engineering Structures 1993.

16. Young, O.C.; Trott, J.J. Buried Rigid Pipes: Structural Design of Pipelines; CRC Press, 1984; ISBN 9780853342359.

17. Thomson, J.; Wang, L. State of technology review report on condition assessment of ferrous water transmission and distribution systems; 2009;

18. Rizzo, P. Water and wastewater pipe nondestructive evaluation and health monitoring: A review. Adv. Civ. Eng. 2010, 2010.

19. Sethna, J. Statistical Mechanics: Entropy, Order Parameters, and Complexity; Oxford University Press, 2006;

20. Shannon, C.E. A Mathematical Theory of Communication. Bell Syst. Tech. J. 1948, 27, 379-423.

21. Farrar, C.; Worden, K.; Park, G. Complexity: A New Axiom for Structural Health Monitoring?; 2010;

22. Farrar, C.; Manson, G.; Worden, K.; Farrar, C.R.; Park, G. The Fundamental Axioms of Structural Health Monitoring . Proc. R. Soc. 2007, 463, 1639-1664.

23. Standards, B. BS 5911-1:2002+A2:2010 Concrete pipes and ancillary concrete products. Specification for unreinforced and reinforced concrete pipes (including jacking pipes) and fittings with flexible joints (complementary to BS EN 1916:2002) 2002.

24. Di Prabhata; K. Swamee; Ashok K. Sharma Design of Water Supply Pipe Networks - Prabhata K. Swamee, Ashok K. Sharma - Google Libri; John Wiley \& Sons, 2008;

Publisher's Note: MDPI stays neutral with regard to jurisdictional claims in published maps and institutional affiliations.

(C) 2020 by the authors. Submitted for possible open access publication under the terms

and conditions of the Creative Commons Attribution (CC BY) license

(http://creativecommons.org/licenses/by/4.0/). 